\title{
The effects of size and color cues on the delayed response of very young children
}

\author{
MARVIN DAEHLER, DANUTA BUKATKO, KATHY BENSON, and NANCY MYERS \\ University of Massachusetts, Amherst, Massachusetts 01002
}

\begin{abstract}
Sixteen children each at 18,24, 30, and 36 months of age participated in a four-choice delayed reaction task to assess memory for location of a hidden object. On one-third of the trials, only place cues were available; on another third, size cues were added; on the remaining third, color cues were added. All age groups benefited from added visual cues on the test trials, with size cues being particularly facilitating. At the end of the test trials, two additional trials were presented in which the size or color cues were surreptitiously switched so that they were no longer redundant with place cues. Only the oldest children were likely to respond directly to a visual cue when in conflict with place cues. The results support a developmental model suggesting a shift from reliance on place cues, to greater reliance on other associated visual cues, in memory for locating a hidden object.
\end{abstract}

Research on the development of object permanence has indicated that children as young as 18 months of age have little difficulty immediately retrieving an object hidden in one of several alternative locations. When a delay is introduced, however, performance is far from perfect, even among children several years older (i.e., Hunter, 1913; Weisberg, 1970). The present experiment, utilizing a delayed response task, was concerned with whether added discriminable cues associated with each of several possible locations for a hidden object benefit memory in very young children and whether such added cues at some point in development may actually dominate search for the hidden object.

Previous experiments have failed to find that very young children effectively use discriminative visual cues to remember the location of a hidden object. Babska (1965) required children to remember which of four boxes contained a toy; each box possessed a distinctive picture on its lid. Children under 3 years of age performed poorly. Loughlin and Daehler (1973) found that the addition of discriminable pictures to place cues also failed to facilitate performance of 27 . and 33-month-olds on a delayed reaction task. On the other hand, 42-month-olds did better when picture cues were added than when place alone could be used to find the hidden object.

It is somewhat surprising that very young children fail to benefit from picture cues, but perhaps they do not conceptually link such cues to the hidden object and its location. Preschoolers do not physically

This research was supported by a grant from the Spencer Foundation to Marvin Daehler and Nancy Myers. We are grateful to Daniel Anderson and Marion Perlmutter for their reading of previous drafts and to Jerome Myers for statistical consultation. Requests for reprints should be sent to Marvin Daehler. Department of Psychology, University of Massachusetts, Amherst. Massachusetts $01(0) 2$. rearrange available supplementary cues to aid memory for the location of an object (Corsini, Pick, \& Flavell, 1968; Flavell, 1970; Ryan, Hegion, \& Flavell, 1970), and still younger children may not establish such linkages conceptually even when the discriminative cues are physically provided. On the other hand, very young children may be able to utilize discriminative visual cues under some conditions. For example, when the visual discriminative cues constitute stable nonarbitrary characteristics defining each location, performance may be improved relative to a condition in which only place cues are available. Cues consisting of dimensions of the boxes themselves, i.e., their size or color, were varied in the present experiment to test this possibility.

Another major question in this experiment concerned developmental differences in the dominance of visual vs. place cues in searching for an object. Babska (1965) and others have proposed that during the 2 nd or 3 rd year of life, visual cues supersede place cues in importance for locating a hidden object. Miller (1934) conducted a series of delayed-response experiments in which boxes differing in color were surreptitiously repositioned during the delay period so that place and color cues were in conflict with regard to location of the hidden object. Although few subjects under 3 years of age were included, the results suggested that children below this age responded primarily to place cues while older children were more likely to use color to govern search. In the present experiment, two similar conflict trials were presented at the end of the task, and it was anticipated that older children would be more likely to use size or color cues rather than place cues in searching for the object. An additional developmental question, however, concerned the relationship between the facilitative effects of visual cues on memory and choice of such cues on conflict trials. 
Would reliance on visual cues on conflict trials and facilitation of memory from added discriminative visual cues be found at about the same age or would facilitation of memory appear considerably earlier than search governed primarily by visual cues?

\section{METHOD}

\section{Subjects}

Eight boys and eight girls, each at mean ages 18, 24, 30, and 36 months $(N=64)$, participated in this experiment. All subjects were within 2 months of their designated age level. Two boys, one at 24 months and the other at 30 months of age, were replaced because of failure to complete all trials.

\section{Apparatus and Materials}

Except for differences in the boxes, the apparatus was identical to that used by Loughlin and Daehler (1973). Basically, it consisted of a felt-covered platform on which four file-card boxes were placed. Located near the front of the platform was a rigid transparent plastic screen which could be raised and lowered manually by the experimenter. The front edge of the platform, screen, and array of boxes were shaped in a concave fashion so that a child. sitting within a semicircular open space. could easily reach each box.

Three different sets of four boxes were used. The control set consisted of four boxes identical in size, $11.3 \times 16.5 \times 10.3 \mathrm{~cm}$, each covered with white contact paper. A color set of the same size was also used. but each box was covered with contact paper of a different color. i.e., red, green, yellow, and blue. The size set varied in size: the boxes were $8.9 \times 3.3 \times 8.3,11.3 \times 16.5 \times 10.3$, $14.5 \times 21 \times 12.3,17.8 \times 24 \times 21.6 \mathrm{~cm}$, respectively, and each box was covered with white contact paper. A curtain located to the rear of the platform screened the unused sets and a second experimenter who arranged the sets of boxes between trials.

Identical brown spools were attached to the front of the lid of each box to aid in its opening. The boxes were positioned $14 \mathrm{~cm}$ from the front edge of the platform and $8 \mathrm{~cm}$ behind the transparent screen. The midpoints of each box were $25.4 \mathrm{~cm}$ apart. One of three squeak toys served as the hidden object. In addition, a small balance toy was used to occupy the child during the delay interval.

\section{Procedure}

After becoming acquainted with the experimenters in a playroom. each child was invited into the nearby experimental room. A parent usually accompanied and sat to the right and rear of each child.

The subject was initially given practice trials in which to find the object immediately after it was hidden. With the transparent screen in position in front of the subject, the experimenter placed a toy into one of the four open boxes while encouraging the child to watch. The experimenter pointed and said "Remember. he's hiding in this one." All boxes were closed (the baited one last), the transparent screen was raised without a delay, and the child was instructed to open the box containing the toy. When correct responses were made. the experimenter provided reinforcing verbal comments and the subject was permitted to withdraw the object and play with it for a short time. If an incorrect response occurred, the child was encouraged to continue opening boxes until the object was located. All subjects were given a minimum of three practice trials with the control set of boxes during which the toy was randomly placed in the boxes. Subjects were required to respond correctly on two of the three trials before being given test trials. A few subjects scattered among the various age groups received an extra trial to reach this criterion. Only one child ( 30 months) required more than one additional training trial.

Twelve test trials and two conflict trials were presented immediately after training. A $25-\mathrm{sec}$ delay was used on each of these trials. During this deiay, the child's chair was rotated $180 \mathrm{deg}$ and the child was encouraged to place rings on a balance toy. At the end of the delay period, indicated by a short tone, the child was returned to his position in front of the apparatus and encouraged to open the boxes.

Each of the sets of boxes was presented once in every block of three test trials. The order of trials was randomized with the restriction that a different set of boxes was presented on successive trials. A different order of trials was given to every subject within an age group. Each box within a set occurred in the same position on all trials for a child, but location of each box was randomized across children. The object was hidden once in every position for each set of boxes and once in each position in every block of four trials and was never hidden in the same position twice in succession.

At the end of the test trials, two conflict trials were presented, one involving the size set and the other involving the color set. The order of these two conflict trials was counterbalanced across subjects. On these trials, the toy was hidden in either the left- or the rightmost box, and during the delay the two end boxes were surreptitiously switched so that place cues were no longer appropriate, and visual cues were no longer redundant with position.

\section{RESULTS}

\section{Test Trials}

Both the percentage of errorless trials and the number of errors per trial were analyzed, but since both measures revealed essentially the same findings, only the results for the first measure are reported. The mean percentage of errorless trials as a function of age and condition are shown in Table 1 . Performance was significantly above chance $(25 \%)$ for each set of boxes at each age level $(\mathrm{p}<.01)$. Moreover, performance on the delayed reaction task improved with age and when color and size cues were available, although the effect of color was somewhat less than size.

An analysis of variance carried out on these data revealed a significant main effect for age $[F(3.56)=9.42, \quad p<.001]$ and cue condition $[F(2,112)=12.80, \quad p<.001]$. Bonferoni $t$ tests indicated significantly more errorless trials for the three older groups than for the youngest group (all EWs <.05), but the percentage of errorless trials for the three older groups did not differ significantly from each other. Bonferoni t tests also indicated that there were significantly more correct trials with the size set than with the color set $(\mathrm{EW}<.05)$ and significantly more correct trials with either of the varying sets than

Table 1

Mean Percentage of Errorless Trials

\begin{tabular}{ccccc}
\hline & \multicolumn{4}{c}{ Cue Conditions } \\
\cline { 2 - 5 } $\begin{array}{c}\text { Age } \\
\text { Groups }\end{array}$ & Control & $\begin{array}{c}\text { Color } \\
\text { Cues }\end{array}$ & $\begin{array}{c}\text { Size } \\
\text { Cues }\end{array}$ & $\begin{array}{c}\text { Combined } \\
\text { Over } \\
\text { Conditions }\end{array}$ \\
\hline 18 months & 39.00 & 40.50 & 53.00 & 44.17 \\
24 months & 48.50 & 64.00 & 68.75 & 60.43 \\
30 months & 53.00 & 64.00 & 73.50 & 63.50 \\
36 months & 61.00 & 76.50 & 84.50 & 74.00 \\
Combined & 50.38 & 61.25 & 69.94 & \\
Over Ages & & & & \\
\hline
\end{tabular}


with the control set $(\mathrm{EW}<.01)$. The Age by Cue Condition interaction failed to be significant. Also, performance did not change over the session; trial blocks was not a significant source of variance.

\section{Error Analyses}

The data were additionally examined for evidence of age-related position, size, and color biases. Fewer correct trials occurred when the object was hidden in the two middle positions, but this was true for only the two older groups. Younger children did less well on trials in which the object was positioned on the right side of the array, suggesting a left position bias in their search activity. A significant Age by Position interaction $[\mathrm{F}(9,168)=2.38, \mathrm{p}=.05]$ in an analysis of variance of position responses confirmed this age difference.

Since the object was hidden only once in each of the four boxes within a set for each subject, nonparametric statistics were used to analyze differences for size and color boxes. However, Cochran $\mathrm{Q}$ tests failed to reveal that some color or size boxes were more likely to be correctly selected than others at any of the four ages or for all subjects combined.

Analyses were also carried out to assess any tendencies to search the location that was correct on the preceding trial. Such errors could be expected by chance on one-third of the incorrect trials. Although $43 \%, 57 \%, 46 \%$, and $40 \%$ of the errors belonged in this category for the youngest to oldest groups, respectively, the frequency of this type of error only differed significantly from chance for the 24- and 30 -month age groups $(\mathrm{p}<.05)$.

\section{Conflict Trials}

Two analyses were conducted to assess the effect of the conflict trials. In the first, the proportion of initial responses to the location cue on the first conflict trial was compared to the proportion of correct responses on the two size and two color trials in which the end positions contained the hidden object. The proportion of correct choices on the four conventional test trials was $.42, .69, .75$, and .92 for the youngest to oldest age groups, respectively. However, on the conflict trials the proportion of location choices were $.19, .31$, .38 , and .44 , respectively. At all four age levels, the proportion of location choices was significantly less on the conflict trial (all ps $<.05$ ). Thus, subjects at every age were less likely to select the end location when visual cues were no longer redundant with place.

The second measure was concerned with the percentage of subjects at each age who responded first to the color or size cue when it conflicted with the place cue. These percentages are shown in Table 2. Order of the color and size conflict trials has been disregarded in this table since there was no evidence to suggest that it had any effect on this measure.
Table 2

Percentage of Subjects Responding First to Size and Color Cues on Conflict Trials

\begin{tabular}{crr}
\hline Age & \multicolumn{2}{c}{ Trial Type } \\
Groups & Color & \multicolumn{1}{c}{ Size } \\
\hline 18 months & 18.75 & 12.50 \\
24 months & 6.25 & 6.25 \\
30 months & 18.75 & 6.25 \\
36 months & 25.00 & 50.00 \\
\hline
\end{tabular}

Thirty-six-month-olds were significantly more likely than subjects in each of the younger groups to begin searching in the box of the correct size (Fisher's exact probability tests, $p<.03$ ), but older subjects were not more likely than younger subjects to initiate search on the basis of the color cue. Thus, in this rather stringent test, after 12 trials in which place cues were always present and always useful, and redundant with visual cues on only some trials, few young children were likely to immediately rely on visual cues when in conflict with place cues. On the other hand, half the 36-month-olds did use size cues to initiate their search.

\section{DISCUSSION}

The results provide evidence that children under 3 years can and do make use of discriminative visual cues to facilitate memory and search for the location of hidden objects. Even the youngest children, only 18 months of age, performed better on trials in which color or size cues were redundant with position. Apparently, size and color are among the properties of containers which help define location of an object, and perhaps the finding that size was the more effective cue indicates that it is the more functional property for these young children. However, further work on dimensional salience, cue scaling, and the role of dimensional, cue, and experiential variation in concept development and problem-solving tasks such as this one, is critical to extending such a line of reasoning (e.g., Brown, Scott, \& Urbano, 1972; Odom \& Corbin, 1973).

The conflict trial data do, however, throw some additional light on the extent to which the children relied on the size and color cues. When the boxes were repositioned during the conflict trials, it was quite clear that while the younger children recognized a change, they did not immediately use the size or color cues as a basis for initiating search. They, like older child ren, were less likely to search at the same location, but they seldom correctly initiated search on the basis of the size and color cues alone. Only the oldest children tested, 36-month-olds, began to initiate search on the basis of these visual cues, and then only when the boxes differed in size.

An interesting finding emerged from the error analysis of this experiment. Subjects tended to search the location correct on the preceding trial. This behavior of initiating search for a hidden object in the place where it was previously found is described as characteristic of children in Piaget's Stage IV. However. Webb, Massar, and Nadolny (1972) reported children as old as 14 and 16 months use this search strategy. The present study surely demonstrated that the predominant search strategy of two- and three-year-olds was to the correct location, yet it is interesting to note that even children who are almost 4 years of age occasionally search the location previously correct.

In summary, all groups demonstrated the ability to perform the spatial localization tasks and did better when discriminative cues were added. While a developmental improvement in performance was observed, all ages showed use of available visual cues when 
redundant with position. When added visual cues were no longer redundant with location, children at all ages appeared to recognize a change. but only the oldest subjects were likely to begin their search on the basis of the visual cues. The data is consistent with a developmental model that describes the child as initially using position to locate objects, perhaps utilizing certain additional redundant defining cues to facilitate memory, and eventually relying on those cues even when in conflict with position cues.

\section{REFERENCES}

BABSKA. Z. The formation of the conception of identity of visual characteristics of objects seen successively. In P. H. Mussen (Ed.). European research in cognitive development. Monographs of the Society for Research in Child Development, 1965. 30 (Serial No. 100), 112-124.

Brown. A. L.. Scott, K. G., \& Urbano, R. C. Psychophysically scaled cue differences. learning rate and attentional strategies in a tactile discrimination task. Journal of Experimental Child Psychology, 1972, 13, 283-302.

Corsini. D. A.. Pick. A. D., \& Flavell, J. H. Production of nonverbal mediators in young children. Child Development. 1968, 39, 53-58.

Flavell. J. H. Developmental studies of mediated memory. In H. W. Reese \& L. P. Lipsitt (Eds.), Advances in Child Development and Behavior (Vol. 5). New York: Academic Press, 1970. Pp. 181-211.
Hunter. W. S. The delayed reaction in animals and children. Behavior Monographs. 1913. 2 (Whole No. 1).

Loughlin, K. A., \& Daehler, M. W. The effects of distraction and added perceptual cues on the delayed reaction of very young children. Child Development, 1973. 44. 384-388.

Miller. N. E. The perception of children: A genetic study employing the critical choice of delayed reaction. Journal of Genetic Psychology, 1934, 44, 321-339.

Odom, R. D.. \& Corbin, D. W. Perceptual salience and children's multidimensional problem solving. Child Development. 1973, 44, 425-432.

Ryan. S. M.. Hegion, A. G.. \& Flavell, J. H. Nonverbal mnemonic mediation in preschool children. Child Development. 1970. 41. 539-550.

WEBB. R. A.. MASSAR, B.. \& NAdOLNy, T. Information and strategy in the young child's search for hidden objects. Child Development. 1972. 43. 91-104.

WEISBERG. P. Delayed response learning in two-year-olds: Gradual vs. sudden changes in the delay interval. Psychonomic Science, 1970, 19, 85-86.

(Received for publication October 14, 1975.) 